$c=2 \cdot 99774 \times 10^{10}$, then $1 / \alpha=h c / 2 \pi e^{2}=136 \cdot 76$ If Rydberg's constant $(109,737 \cdot 43)$ and $e / m(1 \cdot 7589$ $\left.\times 10^{7}\right)$ are substituted in the formula $1 / \alpha=$ $(c / 4 \pi R(e / m) e)^{1 / 3}$, it then becomes $137 \cdot 03$, which is nearly integ al.

Natural Philosophy Laboratory, V. D. HoPPER. University of Melbourne. April 29.

' Laby, T. H., and Hopper, V. D., NATURE, 148, 157 (1939).

${ }^{2}$ Gill, T. P., J. Sci. Inst, 16, 345 (1939).

3 Bäcklin, E., and Flemberg, H., NATURE, 187, 655 (1936).

- Ishida, Fukushina and Suetsuga, Phys. Chem. Res. Tokyo, 32, 57 (1937).

'Fabry, C., and Perot, A., Ann. Chim. et Phys., 13, 275 (1898).

\section{Mass Centre in Relativity}

UNDER this title, in NatURE of April 13, p. 587, Prof. M. Born and K. Fuchs gave some relations between the total and a relative momentum vector of a system of two free particles. They only define the magnitude, not the direction of their relative momentum vector. I believe things become clearer by the following statement. Let $V^{a}, W^{a}(a=0,1,2,3)$ be $(1+3)$-dimensional velocity time-space vectors of the particles $\left(V^{2}=W^{2}=c^{2}\right)$, and $m_{1}, m_{2}$ their scalar masses. Their individual energy-momentum vectors being $i_{a}=m_{1} V_{a}$ and $j_{a}=m_{2} W_{a}$, we may define the total energy-momentum vector by

$$
I_{a}=i_{a}+j_{a},
$$

the relative energy-momentum vector by

$$
S_{a}=\left(m_{2} i_{a}-m_{1} j_{a}\right) /\left(m_{1}+m_{z}\right) .
$$

These definitions entail two identities

$$
\begin{gathered}
\left(m_{1}+m_{2}\right) c^{2}=\frac{I^{2}}{m_{1}+m_{2}}+\frac{m_{1}+m_{2}}{m_{1} m_{2}} s^{2}, \\
0=\frac{2 m_{1} m_{2}}{m_{1}+m_{2}}(I . s)-\left(m_{1}-m_{2}\right) s^{2},
\end{gathered}
$$

which are equivalent to equations (4), (5), (6) of Born and Fuchs.

The total energy-momentum has a time vector character, whereas the relative energy-momentum has a space vector character. The two individual rest masses, being constants of the motion, must be functions of the eight dynamical variables $I_{a}$ and $s_{a}$ :

$c^{2}\left(m_{1}+m_{2}\right)^{2}=I^{2}+2 s^{2}+2 \sqrt{s^{4}+(I . s)^{2}}$,

$c^{2}\left(m_{1}-m_{2}\right)^{2}=I^{2}-2 \frac{s^{2} I^{2}-(I . s)^{2}}{s^{2}+\sqrt{s^{4}+(I . s)^{2}}}$

As regards the centre of mass, which has completely been lost sight of in the communication referred to, I suggest that the proper thing to consider is a centre of inertia rather than a mass centre. The former must be defined with the aid of the bivectors of energy and momentum, $i_{a}-$ or rather inertia and momentum, $i^{a}$-and radius vector, $g^{a}$. The components of this bi-vector are given by the determinants of the matrix

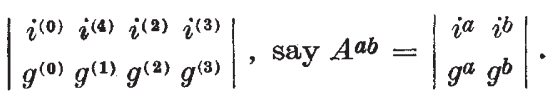

Take the sum of the bi-vectors of two particles, or of any number of particles, and we shall have the bi-vector

$$
M^{a b}=\Sigma_{(n)} A_{n}^{a b},
$$

with six components, of total moment of momentum. Three of these components (for $a=0$ ) define the moment of inertia relative to the origin.

The inertia centre must be defined as the locus of origins in time-space such as to make the moment of inertia vanish. To make the definition invariant, we require the moment of inertia to vanish in a Lorentz frame where the total energy-momentum vector is directed purely parallel to the time axis, so that there is no resultant momentum. Thus

$$
M^{0 b}=0, \quad(b=1,2,3)
$$

In all other frames, that is, for a system of free particles showing resultant momentum, we shall then find a moment of inertia or, say, a polarization of inertia.

For any number of particles, first define $I^{a}=\Sigma_{(n)} i_{n}^{a}$. The determinants

$$
P^{a b c}=\Sigma_{(n)}\left|\begin{array}{ccc}
g_{n}^{a} & g_{n}^{b} & g_{n}^{c} \\
i_{n}^{a} & i_{n}^{b} & i_{n}^{c} \\
I^{a} & I^{b} & I^{c}
\end{array}\right|,
$$

will represent the components of the tri-vector of internal moment of momentum, multiplied by the magnitude of $I$.

The dynamical variables here given were first published in $1927^{1}$, and an illustration of the polarization of inertia is offered in the example of a rolling hoop ${ }^{2}$ and a rotating ring $^{3}$.

Teyler's Stichting,

A. D. FoKKER.

Haarlem.

April 23.

${ }^{1}$ Physica, Ned. T. Natuurk., 7, 330 (1927). Fokker, "Relativiteitstheorie" (Groningen : Noordhoff, 1929)

2 Physica, Ned. T. Natuurk., 1, 35 (1921).

${ }^{3}$ Nuyens, M., Physica, Ned. T. Natuurk., 9, 181 (1929).

We regret having overlooked Prof. Fokker's publications on the subject of the mass centre in relativity. His definition of the centre of inertia does not coincide exactly with our definition of the mass centre. Which definition is to be preferred depends, of course, on the use which can be made of it.

Our purpose was to separate from the Hamiltonian of a system of two particles that term which corresponds to the motion of the system as a whole, and to do it in such a way that the new variables form canonically conjugate pairs. We have shown how this can be done for two free particles by introducing the total momentum $\mathbf{P}$ and the internal momentum $\pi$; the canonically conjugate variables are $\mathbf{R}$ and $\rho$, as mentioned in our previous letter, though we did not give explicit expressions. Here $\mathbf{R}$ is to be interpreted as representing the position of the mass centre.

We have compared the two definitions and found that the mass centre coincides with the centre of inertia in the Lorentz system in which the total momentum vanishes. The two centres are therefore to some extent equivalent.

Although we readily agree that Prof. Fokker's definition is formally much more elegant, we cannot see that it is helpful for the quantum mechanical problem which we had in mind.

Max Born.

KLaUs FUChs.

Department of Mathematical Physics, University of Edinburgh. 